\title{
Les musées scientifiques
}

Tendances actuelles de l'éducation scientifique non formelle

\section{Bernard Schiele}

\section{CpenEdition}

\section{Journals}

Édition électronique

URL : http://journals.openedition.org/ries/3368

DOI : $10.4000 /$ ries.3368

ISSN : 2261-4265

Éditeur

Centre international d'études pédagogiques

\section{Édition imprimée}

Date de publication : 1 juin 1997

Pagination : 105-121

ISSN : $1254-4590$

Référence électronique

Bernard Schiele, "Les musées scientifiques », Revue internationale d'éducation de Sèvres [En ligne], 14 | 1997, mis en ligne le 30 juillet 2013, consulté le 20 avril 2019. URL : http:// journals.openedition.org/ries/3368; DOI : 10.4000/ries.3368

Ce document a été généré automatiquement le 20 avril 2019

(C) Tous droits réservés 


\title{
Les musées scientifiques
}

\author{
Tendances actuelles de l'éducation scientifique non formelle
}

\author{
Bernard Schiele
}

\section{NOTE DE L'ÉDITEUR}

Cet article est la version remaniée de la conférence d'ouverture du colloque Musées et Médias : Pour une culture scientifique et technique des citoyens, organisé par la ville de Genève (Muséum d'histoire naturelle de Genève, Université de Genève, 19-21 mars 1996).

\section{NOTE DE L'AUTEUR}

Je suis redevable à plusieurs collègues du CIRST de certaines idées développées dans cet article. Je voudrais remercier le Professeur Yves Gingras pour les discussions que nous avons eues sur l'histoire des sciences, Monsieur Maurice Landry pour les dossiers et les références qu'il m'a communiqués et le Professeur Camille Limoges (Président du conseil de la science et de la technologie) pour les pistes ouvertes par son travail sur l'avenir des universités.

1 D'entrée de jeu, je donnerai une définition large du musée scientifique ${ }^{1}$. Il est à la fois un lieu de médiation et de négociation d'informations destinées au public ${ }^{2}$.

Médiation : le musée présente des artefacts et des expositions, propose des activités et des programmes. Il organise aussi des événements et des manifestations. Tous ces moyens visent à mettre les visiteurs en contact avec les sciences et les techniques dans tous les domaines. Tout y passe: recherche fondamentale, recherche appliquée, recherche et développement... Il cherche aussi à familiariser les visiteurs avec les idées et les notions clés de certains domaines; à leur montrer des procédés, des applications, des produits ; ou, encore, à les sensibiliser aux effets économiques, sociaux, environnementaux ou éthiques de l'activité des sciences. Chacune de ces médiations fonctionne donc comme une interface et un point de vue sur l'activité scientifique et ses retombées. 
3 Négociation : ces interfaces sont des dispositifs. Et un dispositif n'est jamais amorphe. Il a été pensé et réalisé. Essentiellement, il matérialise un processus de mise en forme de notions, d'idées, de perspectives. Les visiteurs sont donc confrontés à des informations organisées et à des points de vue structurés. Il leur faut donc traiter ces informations, c'est-à-dire : les comprendre, les trier, les classer, les hiérarchiser, etc. Pour ce faire, ils doivent s'orienter dans un complexe de significations et attribuer des «sens » à ce qu'ils voient, entendent ou lisent.

4 Cette définition du musée scientifique est tellement large et englobante, pourrait-on objecter, qu'elle recouvre toute institution qui de près ou de loin connote les sciences, les techniques, les technologies: centre des sciences, centre(s) de culture scientifique technique et industrielle (CCSTI), musée de sciences naturelles, centre d'interprétation, musée de l'automobile ou du chemin de fer. Tous y trouvent leur compte. De plus, elle peut s'appliquer à n'importe quel musée qu'il soit d'art, d'anthropologie, de société, puisque le terme est à la mode, et j'en passe... Tous les musées opèrent des médiations et instituent des espaces de négociation.

5 La question à se poser est alors la suivante : «Quelles sont les formes de médiations et de négociations privilégiées par le musée scientifique?». Car, ce sont ces formes particulières de médiations et de négociations qui le spécifient et le distinguent des autres stratégies muséales (musée d'art, de société, d'histoire, etc.). De plus, les modalités spécifiques de ces formes permettent de distinguer entre elles chacune des institutions muséales qui se réclament du label «science ou technologie». Et, lorsque le musée évolue, ce sont elles qui se transforment par les contenus traités, la façon dont ils le sont, tout comme par les compétences cognitives et sociales requises des visiteurs pour se les approprier.

6 Mais, cette question en soulève immédiatement une autre : «Comment ces formes ontelles évolué ? » Car, pour penser des «tendances », puisque c'est bien de cela qu'il s'agit, encore faut-il pouvoir les rapporter à des institutions et aux changements qui y ont cours.

7 Mon intention n'est pas de brosser une histoire de la muséologie des sciences ${ }^{3}$. Elle n'est pas non plus de décrire en détail chacune des variations de ces dispositifs de médiation et de négociation. Je voudrais simplement présenter quelques-unes des idées-forces autour desquelles s'est cristallisée la muséologie des sciences et des techniques actuelles afin de mieux pointer celles qui, étant émergentes aujourd'hui, vont, me semble-t-il infléchir les discours et les pratiques de diffusion non formelles des sciences et des techniques, et, du coup, les modes de socialisation des sciences et des technologies.

8 J'examinerai deux points: le plateau atteint par la forme actuellement dominante de divulgation non formelle des sciences dans les musées et les facteurs de déstabilisation qui préfigurent l'émergence de nouvelles formes de médiation et de négociation. Toutefois, n'étant pas un adepte de la prospective fiction, je me garderai bien de risquer un pronostic précis et me contenterai d'un diagnostic fondé sur l'examen de trois musées exemplaires le palais de la Découverte, l'Exploratorium et le Science Learning Network. Chacun d'eux, caractérisant beaucoup plus qu'une modalité particulière de divulgation non formelle des sciences, témoigne du mouvement constant de transformation/ recomposition des rapports entre la science et la société et, conséquemment, des modes légitimes de divulgation préconisés à un moment donné. 


\section{Le dispositif muséal actuel}

9 Il y a une quinzaine d'années, la «mort » du musée était annoncée à grand renfort d'études et de colloques. François Dagognet, observateur engagé de l'évolution de la scène culturelle, prédisait le reflux d'une institution, tout entière déployée autour de l'objet, sacralisant la mémoire du passé, tellement prisonnière de son histoire et de ses traditions, qu'elle en était insensible à la mouvance et à la polyphonie sociale. Rébarbative, obtuse, elle s'opposait à toute remise en question: elle n'était plus qu'un 《 cimetière où le visiteur déambulait entre les « tombes » ${ }^{4}$. Non je ne caricature pas ${ }^{5}$ !

10 Avec le recul, ces propos font sourire. Pourquoi? Par le ton, bien sûr! Mais, surtout, parce que se limitant à une critique de surface, ils se faisaient plus l'écho des contradictions qui tiraillaient l'institution qu'ils ne réussissaient à en analyser les véritables causes qui forçaient son évolution. Trois facteurs au moins étaient occultés :

- le fait que le social englobe le muséal ${ }^{6}$;

- qu'une forme muséale dominante n'entre en régression que lorsque des solutions de remplacement répondant à une nouvelle conjoncture en émergence s'offrent à l'usage ;

- que le statut du musée est subsumé sous celui que la société réserve au culturel. Examinons ces trois points en gardant en tête l'objectif de caractériser les idées-forces de la muséologie des sciences actuelle.

\section{Dynamique sociale et dynamique muséale}

11 Une évidence sur laquelle il est toujours utile de réfléchir: le musée, pas plus que l'université ou la recherche, ne vit en vase clos. Il n'est pas insulaire. Il est perméable au social. Ses tiraillements expriment la tension perpétuelle qui lie les institutions à leur environnement et rendent manifeste le nécessaire ajustement de celles-ci à l'évolution mouvante de la conjoncture politique, économique et sociale. Par une sorte d'osmose, le social se rappelle constamment au musée. C'est pourquoi, le réquisitoire passionné de Dagognet, partisan dans ce débat sur l'avenir du musée, d'une part, témoignait des forces qui en profondeur retravaillaient l'institution et, d'autre part, contribuait à asseoir une recomposition du champ muséal, déjà presque achevée et prête à s'afficher publiquement.

12 Laissez-moi illustrer par un exemple le fait que le musée cristallise une conjoncture sociale, et, en conséquence, que l'évolution de cette dynamique entraîne un réalignement des objectifs, des discours et des pratiques de l'institution. Il en va de même pour la diffusion non formelle des sciences: c'est moins la constance d'un tel projet $^{7}$, que sa réaffirmation et sa réactualisation cycliques dans un nouveau pacte social de partage des connaissances, qui importe.

13 Le palais de la Découverte à Paris a longtemps été le fleuron de la muséologie scientifique française. Lors de son ouverture au public, en 1937, il représentait le nec plus ultra de la modernité8. Se pose donc une question : "Quelles ont été les raisons de sa désuétude? ». La vétusté des équipements? Elle peut expliquer en partie l'impression qu'il laissait au visiteur, il y a une quinzaine d'années. Surtout si l'on songe que la nouvelle salle du Système solaire, inaugurée le 19 juin 1984 remplaçait la salle originale... La pauvreté des moyens ? Peut-être ? L'idée de refondre le contenu de la salle remontait à $1970^{\circ}$. Il a donc 
fallu quatorze ans pour y arriver, alors que la durée de vie moyenne d'une exposition permanente est de vingt ans en moyenne. Un mode de conception artisanal ? Un manque d'ouverture aux idées nouvelles? On peut en douter, puisque le palais de la Découverte, dès qu'il a été question d'un nouvel équipement de diffusion des sciences à Paris, a luimême élaboré des propositions. Il me semble que ces raisons, de nombreuses fois évoquées, passent sous silence le véritable argument: il proposait une conception de la science qui ne correspondait plus à la vision que l'on s'en faisait au moment où la Cité des sciences et de l'industrie était en gestation. Le projet de divulgation qu'il incarnait n'était plus en phase avec celui de la société.

14 En quoi le palais de la Découverte se distinguait-il des autres institutions lors de son ouverture $^{10}$ ? «Nous avons d'abord voulu, disait Jean Perrin, familiariser nos visiteurs avec les recherches fondamentales par où s'est créée la science, en répétant journellement les grandes expériences auxquelles ont abouti ces recherches, sans en abaisser le niveau, mais pourtant de façon accessible à un très grand nombre d'esprits. Et nous avons voulu par là répandre dans le public le goût de la culture scientifique en même temps que les qualités de précision, de probité critique et de liberté de jugement que développe cette culture et qui sont utiles à tout homme, quelle que soit sa carrière ${ }^{11}$ . \

Comme tous les musées et tous les centres de science qui ont vu le jour depuis, le palais de la Découverte s'est donné pour mission de diffuser la culture scientifique et de la faire partager. Il entend initier à la pensée scientifique. Pour ce faire, il vise à faire « comprendre la part déterminante que la Découverte a prise dans la création de la civilisation » en réalisant des expositions vivantes « où sont autant que possible, répétées de façon spectaculaire (...) les découvertes fondamentales qui ont élargi notre intelligence (...) assuré notre emprise sur la matière (...) ou augmenté notre sécurité physiologique ${ }^{12}$ " Dans le fond, sa mission est-elle vraiment différente des institutions qui ont été créées depuis ? Laissons cette question ouverte.

En «convenant avec Duby ${ }^{13}$ que la diffusion d'un type de culture s'effectue en synchronisme avec la formation d'un groupe social $»^{14}$, Eidelman a montré que la création du palais de la Découverte a coïncidé avec la professionnalisation de la recherche en France - actualisée par la formation du CNRS. L'affirmation d'une nécessité sociale de la science s'enracine alors dans la conviction profonde que la recherche pure ${ }^{15}$, activité quasi esthétique et motivée par une curiosité gratuite, est une quête désintéressée de l'inconnu qui culmine dans la découverte ${ }^{16}$. C'est cette perspective que le palais de la Découverte va "modéliser" par les modes de médiation qu'il instaure: l'expositionspectacle, les démonstrateurs, l'invitation à toucher et le presse-bouton - ces deux derniers annonçant l'interactivité ${ }^{17}$.

17 Le palais de la Découverte n'est pas un musée-mémoire, c'est un musée-idée. Il est tout entier organisé autour du savoir disciplinaire et des sciences fondamentales ${ }^{18}$. C'est évidemment ce savoir qu'il promeut et dont il valorise la dissémination. Son modèle de médiation repose sur la classe-laboratoire transposée en exposition-spectacle, animée par des démonstrateurs qui reproduisent des expériences spectaculaires et les expliquent à un auditoire. Il s'agit «de fournir aux perceptions de l'individu regardant le monde extérieur, un écran de concepts sur lequel il projette et repère ses perceptions ${ }^{19}$. La relation de celui qui sait à celui qui ne sait pas circonscrit l'espace symbolique de négociation du sens ${ }^{20}$. 

tributaire d'une certaine conception de la science, et des dispositifs de production et d'utilisation des connaissances dominants. Cette vision, répercutée par Jean Perrin, voulant que la recherche fondamentale désintéressée "par un retour singulier » soit à "l'origine de toutes les inventions à caractère utilitaires ${ }^{21}$ domine largement la plus grande partie de notre siècle. Elle a trouvé son programme dans le célèbre rapport de Vannevar Bush, Science, The Endless Frontier, rédigé à la fin de la Seconde Guerre mondiale et dont est sorti l'appareil de recherche fondamentale américain - supporté par l'État et concentré dans les grandes universités.

Le déplacement du centre de gravité, au tournant des années quatre-vingt, qui correspond à la montée des techno-sciences ${ }^{22}$, va entraîner un bouleversement de l'institution muséale. L'arrivée des centres de sciences, avec leur volonté d'ouvrir une fenêtre sur le savoir et le savoir-faire technique et industriel, caractérise cette reconfiguration ${ }^{23}$. En France, la Cité des sciences et de l'industrie incarne ce renversement de tendance.

Donc, puisque le musée scientifique est en quelque sorte un laboratoire social de diffusion, nous garderons en mémoire trois questions: "Quels sont aujourd'hui les nouveaux dispositifs porteurs de production et d'utilisation des connaissances? A quel groupe d'acteurs sociaux se rapportent-ils?» et «Quelles modalités de diffusion impliquent-ils?». Les réponses à ces questions nous renseigneront sur les tendances présentes.

21 Toutefois, avant d'aller plus avant, il nous faut aussi considérer des éléments qui surdéterminent ce déplacement du centre de gravité.

\section{Le développement de l'interactivité}

Au tournant des années quatre-vingt, trois facteurs, au moins, influent sur l'évolution de l'institution muséale :

- la « communication » imprègne tout le discours social ;

- le statut du visiteur change : c'est vers lui que convergent les opérations de médiation du musée ;

- l'interactivité est vue comme le moyen par excellence de réussite de la négociation des contenus par le visiteur.

23 Au moment où le musée-objet était cloué au pilori en Europe, la muséologie scientifique nord-américaine influençait déjà fortement le mouvement de recomposition alors en cours. Elle a marqué de son empreinte presque tous les musées qui se sont ouverts depuis ${ }^{24}$. Notons au passage que, la muséologie scientifique contemporaine a commencé à se mettre en place il y vingt-cinq ans environ : l'Exploratorium et le Centre des sciences de l'Ontario, ouverts l'un et l'autre en 1969, peuvent servir de points de repère.

En faisant du musée un lieu de médiation des connaissances, sollicitant la participation active du visiteur, volontairement situé au centre du dispositif, l'Exploratorium et le Centre des sciences de l'Ontario ont imposé une vision et révolutionné la pratique de la muséologie scientifique. Le succès de la formule qui ne s'est jusqu'à présent jamais démenti a fortement contribué à la pénétration de cette conception. Il fallait visiter le palais de la Découverte et l'Exploratorium - au moment où la Cité des sciences et de l'industrie était encore à l'état de projet - pour saisir tout ce qui opposait ces deux 
approches. Il faut aujourd'hui comparer la Galerie de zoologie du Muséum national d'histoire naturelle ${ }^{25}$ à la Grande Galerie du Muséum, ouverte au public en $1993^{26}$, ou prendre connaissance du programme du futur musée des Arts et Métiers ${ }^{27}$ pour mesurer le changement de perspective qui s'est opérée ${ }^{28}$. En substance, le musée scientifique s'est recomposé autour du visiteur. C'est lui qui maintenant occupe le devant de la scène. Un exemple: trois cent soixante-quatre jours par année, le Centre des sciences de l'Ontario de Toronto ${ }^{29}$ invite le visiteur qui s'y présente à observer et manipuler le plus grand nombre des huit cents artefacts répartis dans plusieurs sections: Technologie, Transports, Communication, Matière, Richesses naturelles du Canada, Énergie, Transformation, Exploration spatiale, etc. Au cours de leur visite, les enfants - et les adultes - peuvent escalader une paroi rocheuse, explorer l'intérieur d'une grotte, s'enfoncer dans une forêt tropicale, composer électroniquement une carte postale, conduire un véhicule spatial, et ainsi de suite. Les écoliers accompagnés d'un instituteur, ont le choix entre trente-six programmes et ateliers destinés aux garderies, aux classes du primaire et aux lycéens. Par exemple, le Jason Project permettait jusqu'à tout récemment d'observer à partir de plusieurs points de vue, en " téléprésence ", les coulées de lave des volcans actifs d'Hawaï qui s'enfonçaient dans le Pacifique et d'interroger en direct le Professeur Ballard qui dirigeait l'équipe sur place; les férus d'exploration spatiale sont invités à participer à une mission lunaire... De plus, des conférences et des événements spéciaux sont organisés chaque jour. Bien sûr, les intéressés peuvent devenir membres du Centre, s'inscrire à des clubs, etc.

Cette polyvalence centrée sur le visiteur n'a rien d'exceptionnel. Elle est caractéristique de l'offre muséale contemporaine, qu'il s'agisse des musées d'art, de civilisation, de société ou de science. Tous tentent de diversifier expositions, programmes, activités et événements de façon à créer un environnement multi-sensoriel et multi communicationnel qui sollicite constamment le visiteur et multiplie les points d'ancrage potentiels. Tout est mis en œuvre pour susciter, stimuler, capter et fixer son attention, le motiver, éveiller son intérêt ou mobiliser ses styles cognitifs. Plus que jamais, les musées ont fait leurs les préceptes de Macluhan ${ }^{30}$ qui soutenait que, pour être réalisés, les objectifs de diffusion requéraient la mise à contribution de tous les moyens médiatiques. Congrûment, il ajouterait certainement aujourd'hui le multimédia et l'Internet à la gamme de ces moyens.

Cette irruption du visiteur n'a été ni fortuite ni soudaine. Quatre groupes de facteurs peuvent être invoqués.

Le premier est lié au développement des industries culturelles, lesquelles se nourrissent de l'idéologie de la communication et dont le poids sur les appareils culturels commence à se manifester dès les années soixante-dix pour ensuite s'amplifier ${ }^{31}$. Cette pression s'exerce de deux façons: par un questionnement du soutien de l'État aux appareils culturels et par l'imposition de critères de performance calqués sur ceux du secteur privé. On pourrait ajouter que cette pression s'exerce au moment même où le musée s'ouvre comme nouveau débouché à une large gamme de services issus du développement des métiers de la communication. Certes, ceci contribue à sa professionnalisation, mais surtout transforme sa façon de faire les choses: le bricolage artisanal est irrémédiablement terminé. Le musée se voit donc contraint de développer une perspective originale pour concurrencer l'offre des industries culturelles: l'idée de la satisfaction du visiteur devient une composante essentielle de la démarche de médiation. 

rapport, To Improve Learning ${ }^{32}$, publié au États-Unis donne le ton: la technologie éducationnelle, grâce à l'audiovisuel - puisque l'autoroute électronique n'était pas encore là ! - convenablement utilisé, intégré systématiquement à l'enseignement programmé, supporté par la recherche en apprentissage et communication augmentera l'efficacité et la productivité de l'école ${ }^{33}$. Notons, au passage, que l'idée du « facilitateur » se substituant à celle de l'enseignant est, pour la première fois mise en application. L'audiovisuel ne tiendra pas ses promesses, mais le programme de la technologie éducationnelle, lui, restera.

Les prémisses de la technologie éducationnelle, si elles ne réussissent pas à transformer l'école américaine, ouvrent néanmoins la porte au rapprochement de l'école et du musée : il devient le laboratoire et la vitrine de l'école transformée. Elles pavent aussi, dans une autre perspective, la voie à une marchandisation de l'enseignement. J'en ai retenu quatre, pour illustrer mon propos. Il est nécessaire :

- d'établir un lien entre l'école et le monde extérieur, dont elle a trop longtemps été coupée, afin de motiver les étudiants en répondant mieux à leurs intérêts individuels ;

- de répondre aux besoins des individus en s'adaptant aux différences qui caractérisent leurs modes d'apprentissage ;

- de contrôler par des méthodes de calcul et d'évaluation efficaces les coûts sans cesse croissants de l'éducation, en leur appliquant des critères de rendement et d'efficacité basés sur la qualité des étudiants formés, en fonction de l'argent, du temps et du talent ;

- de se rappeler que le développement futur des recherches, en particulier celles sur le processus d'apprentissage, dépend directement du développement de la technologie car elle délimite à la fois le cadre problématique et celui des possibilités de mise en application des résultats obtenus ${ }^{34}$.

Le troisième est lié au mouvement des pédagogies dites actives, alternatives, communautaires, coopératives, etc. - Il faut se rappeler que les années soixante-dix sont aussi des années de contestation aux États-Unis et ailleurs.

Toutes ces pédagogies ont en commun de proposer une relation pédagogique telle que l'élève en occupe le centre et maîtrise son propre processus d'apprentissage.

Le quatrième et dernier est lié au développement de l'évaluation dans les musées, dont $\mathrm{C}$. G. Screven s'est fait le promoteur et le défenseur. Celui-ci soutient que l'exposition doit être conçue pour le visiteur en fonction d'objectifs prédéterminés. Il propose un ensemble de mesures tant pour optimiser la relation de communication instaurée par l'exposition que pour renforcer le rôle éducatif du musée, faisant alors l'objet de débats nationaux ${ }^{35}$. J'ajoute que les modes non formels de diffusion et d'apprentissage commencent par le biais de l'évaluation à entrer dans la culture des musées.

Tout ceci montre à l'évidence que le musée actuel, imprégné de ces courants de pensée, est porteur d'un projet pédagogique, structuré autour du visiteur, fondé sur un rapprochement avec l'école, dont il incarne les formes les plus innovatrices sans pour autant s'y assimiler.

35 L'Exploratorium est un bon exemple de l'aboutissement de cette évolution ${ }^{36}$. Il a été créé en 1969 par le physicien Franck Oppenheimer. Depuis sa création, son rayonnement a eu un impact décisif sur l'évolution des activités éducatives dans les musées scientifiques. L'Exploratorium a deux partis pris : l'action et les enseignants.

Revue internationale d'éducation de Sèvres, 14 | 1997 

véritable "connecteur", qui stimule l'intérêt, la curiosité, l'intelligence et fait comprendre à l'utilisateur qu'il a la capacité de découvrir et de comprendre. Les artefacts, présentant des phénomènes physiques, sont donc conçus pour provoquer une interaction. Ils sont là pour être touchés, expérimentés et manipulés. Mais cette manipulation est suffisamment contraignante "pour soutenir l'intérêt d'explorer un phénomène principal, et un seul à la fois, et suffisamment ouvert pour s'accommoder de plusieurs démarches. C'est un design qui conduit surtout à se poser des questions et à réaliser qu'une question mobilise obligatoirement une partie de notre propre savoir qui sort de l'état inerte $»^{37}$. Du coup, apprendre, c'est aussi, découvrir les vues des auteurs et des concepteurs sur la nature, leur culture et plus précisément leur «culture d'apprendre ". "Ce sont des outils d'initiation soulignant le caractère social de l'apprentissage. Ils font progressivement découvrir que chacun peut devenir membre de cette communauté humaine qui essaie de comprendre la nature et lui donne du sens ${ }^{38}$. »

Corollairement, la connaissance du comportement du public et des stratégies cognitives qu'il mobilise dans un environnement non formel est essentielle pour la conception de dispositifs efficaces ${ }^{39}$. Aussi, les recherches menées à l'Exploratorium se consacrent-elles uniquement au visiteur: l'enjeu est la connaissance du visiteur en situation d'apprentissage non formelle. Notons au passage que c'est dans cet esprit que l'Exploratorium fabrique et teste lui-même les artefacts qu'il conçoit. est simple : si l'on familiarise suffisamment les enseignants avec les sciences et qu'on les dote de ressources pour asseoir leur confiance ${ }^{41}$, ils peuvent alors propager ce double sentiment de familiarité et de confiance. La formation est centrée sur la démarche individuelle d'apprentissage. Les questions et interrogations guident le déroulement des cours. Il faut amener chaque enseignant à expérimenter quand il ne comprend pas et à découvrir les choses de lui-même. Cette formation repose uniquement sur les artefacts de l'Exploratorium.

39 Avant d'en terminer avec l'Exploratorium, je voudrais mentionner les explainers - les «expliquants». Oppenheimer a emprunté au palais de la Découverte l'idée de démonstrations conduites par des étudiants. Les « expliquants » sont des lycéens formés par le musée à l'entretien des artefacts et à l'explication de leur fonctionnement. La première idée est de convier des jeunes à s'adresser au public jeune et adulte pour, à la fois, faciliter la prise de contact et optimiser les échanges. La seconde, est de tisser des liens avec les écoles, puisque ces guides ne sont admis au programme de formation que sur la recommandation de conseillers pédagogiques. La troisième est de démontrer, par l'assurance dont ils témoignent, que la science est accessible à tous ${ }^{42}$. Enfin, notons qu'une fois formés et engagés, ce sont eux qui assurent en plus des démonstrations toutes les fonctions d'accueil et d'encadrement des visiteurs.

\section{Les pratiques muséales émergentes}

40 On pouvait raisonnablement espérer, après un tel réalignement de la mission qu'un plateau serait atteint ${ }^{43}$; qu'une période d'équilibre dynamique permettrait, en partie grâce aux ressources attribuées à chaque institution, d'explorer à loisir les potentialités offertes par ce renouvellement de perspective. Seulement voilà... La muséologie 
scientifique - et plus généralement la muséologie tout court - est de nouveau entrée dans une période d'évolution rapide et profonde. Prenons rapidement le pouls de la situation présente.

\section{Diminution des ressources}

41 La croissance accélérée que les économies occidentales ont connue depuis la fin de la Seconde Guerre mondiale, aiguillonnée par la guerre froide et le miracle japonais, entrecoupée certes de crises, de stagnations et de reprises, marque aujourd'hui le pas. Ce tassement, annoncé depuis plusieurs années, est maintenant une réalité malgré le mouvement de globalisation et de mondialisation de l'économie. Et les mesures de relance traditionnelles semblent sans effet. L'endettement des États plafonne leur capacité de dépenser ${ }^{44}$. On observe donc de leur part un mouvement généralisé de retrait, ou de profonde réorganisation des domaines d'intervention dont, il y a peu de temps encore, ils se percevaient comme les maitres d'œuvre naturels et les garants. Aux ÉtatsUnis et au Canada, l'éducation, la recherche, la santé, les programmes sociaux et la culture sont fortement touchés. L'Union européenne entre aussi dans cette spirale. C'est bien une période d'incertitude qui s'installe pour la culture, et, évidemment, pour la muséologie scientifique. Il va falloir apprendre à gérer la décroissance sur une longue période. Ceci n'est pas une tendance, mais un état de fait! Notons, par ailleurs, que les musées ont appris ces dernières années que le mécénat, toujours ponctuel, ne pouvait combler un manque à gagner, surtout s'il devient systématique. Les musées vont devoir apprendre à travailler différemment.

\section{Intégration de l'école et du musée}

Ils vont devoir surtout apprendre à travailler d'une manière qui rencontre les besoins émergents. Car le mouvement de fond qui se dessine, à la fois, désigne de nouveaux lieux de production de connaissances, un nouveau rapport aux connaissances et un nouveau mode de production de ces connaissances ${ }^{45}$.

Tout d'abord le contexte de production des connaissances : celles-ci tendent de plus en plus aujourd'hui à être produites en contexte d'application, ceci signifie que la recherche « ne cesse de résoudre des problèmes théoriques (...) mais dans une visée de réalisations pratiques ». Ceci implique une approche structurellement transdisciplinaire mais aussi une diversité et une hétérogénéité des "dispositifs organisationnels engagés dans la production de connaissances $»^{46}$. Des lieux, des formes d'organisation nouvelle émergente et des partenaires se manifestent: il s'ensuit que les résultats ne sont plus mesurés uniquement à l'aune des chercheurs. D'autres critères d'évaluation interviennent. Mais, quelle est l'incidence de ce bouleversement sur le musée de science ? Ni la référence à la découverte et la recherche fondamentale ni la valorisation des applications des technosciences ne suffisent à illustrer ce contexte de production et d'échange de connaissances.

Le modèle de transmission des connaissances initié par le palais de la Découverte pas plus que l'accent sur l'interactivité, le hands on exhibit en jargon du métier, ne rencontrent, au plan de la médiation, les habiletés cognitives et sociales requises pour s'insérer dans ce nouvel environnement. De plus, dans la mesure où la recherche en équipe multidisciplinaire prend le pas sur la démarche individuelle, même une approche muséale 
centrée sur le visiteur «maître » de son apprentissage est insuffisante. Puisqu'il faut maintenant apprendre à produire collectivement des connaissances, c'est-à-dire développer des habiletés socio-cognitives de réflexion, de création et de travail qui se manifestent en groupe, le musée devra mettre en avant ces modes de négociation du rapport aux savoirs.

Examinons une piste qui se dessine. L'Exploratorium de San Francisco, le Miami Museum of Science, le Museum of Science de Boston, l'Oregon Museum of Science and Industry de Portland, le Science Museum of Minnesota de St.-Paul et le Franklin Institute Science Museum de Philadelphie ont créé avec l'aide de la compagnie Unisys Corporation et de la National Science Foundation le Science Learning Network (SLN Alliance) ${ }^{47}$. L'objectif est d'intégrer les ressources éducationnelles de chacun de ces centres de sciences grâce au Web afin d'offrir des nouveaux moyens d'enseignement et d'apprentissage des sciences ${ }^{48}$. Le projet remonte à 1993, au moment où l'Internet permettait principalement d'échanger du texte, comme le courrier électronique et l'expédition/réception de dossiers, de communiquer à distance avec un autre ordinateur et de consulter des d'informations (banques de données) stockées dans des milliers d'ordinateurs. Pour le directeur du projet, Steve Baumann, ce n'était pas suffisant : « avoir accès à la science ne suffisait pas, il fallait y participer. Nos documents, ajoute-t-il, ne sont pas que du texte, ils comportent des sons, des images, de la vidéo, des interactifs ». Qui plus est, "nous privilégions les questions sur les réponses et croyons que la démarche de recherche de solutions est aussi valable que la solution obtenue ${ }^{49}$ ». L'arrivée du Web (World-Wide Web) avec ses immenses potentialités et surtout sa simplicité d'utilisation a permis le lancement du projet. Ces musées ont donc entrepris de partager leurs ressources et leurs expertises ${ }^{50}$ pour fournir, dès 1998, aux instituteurs de science de la documentation, online resources, et de l'aide professionnelle en téléinformatique. De plus, comme chaque musée est associé à une école participante, affiliée au programme, celle-ci servira d'école locale de démonstration en ligne. Une bibliothèque électronique s'adressant aux mordus des sciences leur facilitera une exploration, (WWW explorers), active et stimulera la publication, (WWW publishers) ${ }^{51}$. Tout ceci requiert le développement de logiciels pour simplifier les tâches requises pour les écoliers comme pour les enseignants.

Cet exemple montre quelle orientation commence à se dessiner. Il s'agit moins de s'approprier individuellement des connaissances que d'apprendre à résoudre collectivement des problèmes en mobilisant des compétences individuelles et en déterminant collectivement les stratégies et les moyens pour augmenter la performance du groupe. L'informatique est évidemment un outil incontournable. Une remarque au passage : le musée virtuel n'est pas celui qu'on croit ! Ce n'est pas le musée que l'on visite ou dont on consulte le programme d'activités à partir de chez soi. Même si cela est bien amusant. Le musée virtuel est celui qui intègre les fonctions et les services et qui les lie entre eux et à leur environnement scolaire, communautaire ou autre. On saisit immédiatement, du coup, les implications économiques (coûts d'infrastructure, d'expertise, d'installation et de maintien d'un lien téléinformatique) et pédagogiques, par la recomposition des activités associées à l'enseignement, à la préparation du matériel didactique, à l'apprentissage et à l'évaluation de celui-ci ${ }^{52}$. On voit le formidable banc d'essai que cela représente pour tout le marché potentiel de l'école et de la culture. L'intégration potentielle de l'éducation formelle et de l'éducation non formelle dans l'espace de la communication généralisée n'est plus une utopie. Et l'arrimage d'institutions aux missions initialement distinctes, comme l'école et le musée, s'envisage 
tout naturellement, mais à partir d'un lieu qui n'est plus ni celui de l'école ni celui du musée. On comprend mieux dans cette perspective pourquoi la recherche sur la divulgation non formelle des savoirs connaît actuellement un développement considérable ${ }^{53}$.

47 Certes, certaines formes actuelles se maintiendront. Les musées de science présenteront encore des expositions, organiseront des ateliers, des événements spéciaux, produiront du matériel didactique... Il est évident que les institutions dotées de collections importantes, comme les musées de sciences naturelles, continueront de les mettre en valeur. Mais, il faut se demander comment les modes de médiation «interactifs" résisteront à l'arrivée de l'autoroute électronique (Electronic Higway) et à la montée des nouveaux usages car le recours à la simulation ouvre des perspectives considérables ${ }^{54}$.

Qu'adviendra-t-il, dans un contexte de rareté et de redéploiement de l'institution, du visiteur, tel qu'on le conçoit aujourd'hui? Il est là pour rester, car il représente un ajustement structurel trop important de l'institution muséale contemporaine pour être relégué à un rôle secondaire. Toutefois, n'embrayant que sur certaines médiations et négociations, les nouvelles fonctions qui progressivement se mettent en place constitueront leurs usagers. Ce qui devrait contribuer à segmenter encore plus les publics des musées, suivant en cela le mouvement que l'on observe dans les médias et dans les services.

Voilà, en substance ce que je pense être les tendances porteuses. Je me suis limité à quelques exemples, car j'ai cherché à dégager la dynamique qui reconfigure actuellement le musée scientifique et les pratiques de divulgation non formelle des sciences. J'ai aussi voulu montrer que le musée se structurait autour d'une conception de la science et de certains modes sociétaux de production et de diffusion des connaissances, et, en conséquence, que l'évolution de ceux-ci transformait les dispositifs de médiation et les processus de négociation par lesquels les sciences $d u$ réel deviennent sciences dans le réel 55 .

Une remarque finale : on accorde d'emblée aux nouvelles technologies, dites fort à propos de communication, un effet de renforcement de cette dynamique d'ensemble. Elles explorent constamment de nouveaux territoires que l'on peut annexer et exploiter. Tout se passe comme si chaque innovation libérait un potentiel tel d'enrichissement de la relation de médiation de connaissances, instaurée par le musée scientifique, qu'y renoncer, dans l'effervescence technologique actuelle, équivaudrait à se priver des moyens susceptibles d'optimiser la mission de diffusion de l'institution, c'est-à-dire accepter de s'appauvrir volontairement. Or, mon propos était justement de souligner que les nouvelles technologies de communication semblaient d'autant plus révolutionnaires qu'elles ancraient des pratiques déjà actualisées dans le social.

\section{NOTES}

1. J'écris musée scientifique pour faire court, car il est bien évident aujourd'hui que le musée scientifique est aussi et indissociablement, technologique. 
2. Il est aussi, bien évidemment, un des lieux de socialisation de ces mêmes savoirs.

3. Une présentation, même sommaire de l'évolution récente de la muséologie des sciences dépasse le cadre de cet article. Pour un survol historique, voir : K. Hudson, Museums of Influence, New York, Cambridge University Press, 1987.

4. F. Dagognet, Le musée sans fin, Seyssel, Champ Vallon, 1984, passim.

5. En toute honnêteté, il me faut préciser que Dagognet proposait quelques pistes susceptibles d'amorcer une sortie de crise. F. Dagognet, 1984, op. cit. ; F. Dagognet, «Quel musée demain? ", in : J. Hainard, et R. Kaehr, (éd.), Temps perdu, temps retrouvé, Neuchâtel, Musée d'ethnographie, 1985, p. 99-109.

6. Ceci n'implique pas un déterminisme sociétal: la recherche sur les organisations (des structures) a montré que celles-ci réagissent aux pressions de la société (un complexe de structures) en fonction de leurs propriétés qui les définissent comme des totalités et assurent leur autoréglage. Organisations et société sont donc en tension dynamique. Pour un survol de la question: J. Piaget, Le structuralisme, Paris, Presses universitaires de France, 1974 ; pour les organisations: H. Mintzberg, The Structuring of Organisations, Englewood Cliffs, Prentice-Hall, 1979.

7. C'est parce que l'on manque généralement de recul historique en cette matière que l'on croit que le souci d'une culture scientifique partagée est récent. Pour une mise en perspective historique, voir pour un survol : D. Raichvarg, J. Jacques, Savants et Ignorants - Une histoire de la vulgarisation des sciences, Paris, Seuil, 1991 ; B. Schiele et al, « Le Québec : historique de la culture scientifique et technologique et bilan de l'action gouvernementale », in : B. Schiele (éd.), Quand la science devient culture, Québec, Éditions MultiMondes, Lyon, Centre Jacques Cartier, 1994, p. 13-86. 8. On comprend que le palais de la Découverte va se distinguer radicalement de la muséologie du Musée national des techniques et du Muséum national d'histoire naturelle dont les modes de médiation sont les séries.

9. Pour l'analyse de cette salle et une mise en contexte de sa conception voir: B. Schiele et L. Boucher, «Une exposition peut en cacher une autre », in : B. Schiele, C. Perraton, L. Boucher, Ciel une expo. Approche de l'exposition scientifique, Paris, Expo Media, 1987, p. 67-222.

10. Pour une problématisation de l'évolution de la muséographie, voir: C. Perraton, «Voir et toucher la science. Éléments pour l'analyse des stratégies communicationnelles à l'œuvre dans les musées de science et de technologie ", in: B. Schiele, C. Perraton, L. Boucher, Ciel une expo. Approche de l'exposition scientifique, Paris, Expo Media, 1987, p. 15-66.

11. Jean Perrin, cité par A. J., Rose (1967), « Le palais de la Découverte », Museum, 20 (3), p. 206.

12. Jean Perrin, cité par M. Roussel, Le public adulte au palais de la Découverte (d'après les principaux résultats d'une enquête sociopédagogique, 1970-1978), Paris, Palais de la Découverte, ronéotypé, 1979, p. 2.

13. G. Duby, «La vulgarisation des modèles culturels dans la société féodale », in: M. David et al., Niveaux de culture et groupes sociaux, Actes du colloque, Paris, Mouton, 1967.

14. J. Eidelman, «Culture scientifique et professionnalisation de la recherche », in : D. Jacobi, et B. Schiele, Vulgariser la science. Le procès de l'ignorance, Seyssel, Champ Vallon, 1988a, p. 175.

15. Il faudrait pouvoir rappeler comment s'est constitué et a pris forme l'idée de "science pure » autour de laquelle tout le travail de diffusion du palais de la Découverte va se concrétiser. Malheureusement ceci dépasserait largement le cadre de cette conférence. Je voudrais toutefois souligner que si le palais de la Découverte marque une rupture, l'idée de la mise en spectacle de la science pure était dans l'air du temps. L'exposition Faraday (Londres, 1931), l'Exposition internationale de Chicago: A Century of Progress 1933 (1933-1934), avaient, parmi d'autres manifestations, contribué à baliser le terrain. Notons au passage que les Expositions universelles ont, dans l'ensemble, très largement présenté les techniques et l'industrie. Voir sur ces deux points : R. W. Rydell, World of Pairs. The Century-of-Progress Exposition, Chicago, The University of Chicago Press, 1993.

Revue internationale d'éducation de Sèvres, 14 | 1997 
16. J. Eidelman, La création du palais de la Découverte (Professionnalisation de la recherche et culture scientifique dans l'entre-deux guerres), thèse, Paris, Université de Paris V-René Descartes, ronéotypé, $1988 \mathrm{~b}$, passim.

17. J. Eidelman, op. cit., 1988a, p. 178.

18. $\mathrm{Y}$ compris son organigramme et, a fortiori, le processus de conception et de réalisation des expositions. Sur ce point voir : H. Denis, L'organisation du palais de la Découverte, Montréal, École Polytechnique, ronéotypé, 1988a.

19. A. A. Moles, Sociodynamique de la culture, La Haye, Mouton, 1967, p. 28.

20. Le préambule au projet de palais de la Découverte précise: «(d)es démonstrateurs (avec disques phonographiques et films cinématographiques) donneront les explications nécessaires. De brefs commentaires sous forme de tableaux relieront logiquement les expériences et formeront pour chaque science un ensemble logique et indiqueront quelles inventions ou applications pratiques ont jailli de chaque découverte.» Cité par J. Eidelman, op.cit., 1988a, p. 180.

21. J. Eidelman, op. cit., 1988a, p. 45.

22. Voir : P. Papon, Les logiques du futur, Paris, Aubier, 1989.

23. L'exemple du Québec est particulièrement instructif à cet égard: l'analyse des projets de musée scientifique - sans qu'il ne voie le jour - qui se sont succédés, presque sans interruption depuis 1976, au moment où le gouvernement québécois entreprend de doter la Province d'une politique culturelle, montre clairement le déplacement du discours vers le pôle technoscience. Le dernier projet en date accentue encore cette tendance. Ministère de l'Industrie, du Commerce, de la Science et de la Technologie, Proposition de concept pour le Musée des sciences et des technologies du Québec, ronéotypé, 1995 ; B. Schiele. et C. Tarpin, «La recomposition du champ muséal au Québec ", in: B. Schroeder-Gudehus, La Société industrielle et ses musées. Demande sociale et choix politiques : 1890-1990, Paris, éditions des archives contemporaines, 1992, p. 253-269.

24. Évidemment les influences et les échanges sont complexes. Une analyse plus fine devrait tenir compte du mouvement de l'écomuséologie et de l'arrivée des musées de société ou de civilisation ainsi que de leur influence respective. Toutefois, il me semble, qu'ils ont en commun d'optimiser la communication avec le visiteur.

25. Pour ce faire une idée de ce qu'elle était avant sa fermeture au public, voir : J. Kreienbühl, Le monde merveilleux de la galerie de zoologie, Bâle, Galerie Specht, 1988.

26. Voir notamment: Cellule de préfiguration - directeur Michel Van Praët, La Galerie de l'Évolution, concepts et évaluation, Paris, Muséum national d'histoire naturelle, 1991.

27. D. Ferriot, B. Jacomy, «Le musée des Arts et Métiers. Problématique d'une rénovation », in : B. Schiele, E. Koster (éd.), Science Museums for the Next Century, à paraître.

28. Je passe volontairement sous silence la muséologie nord-américaine, car sa présentation entraînerait un développement, lequel, faute de temps ne peut malheureusement être présenté aujourd'hui. Je m'en tiendrai donc aux réalisations utiles à mon propos. Pour une présentation d'ensemble du cadre, de l'organisation et de l'administration des centres de science et de technologie, voir : V. J. Danilov, Science and Technology Centers, Cambridge, The MIT Press.

29. Il est possible pour les intéressés qui voudraient en savoir plus de se faire une idée des activités offertes en composant : <http://www.osc.on.ca/>

30. M. MacLuhan, H. Parker, J Barzun, Exploration of the Ways, Means and Values of Museum Communication with the Visiting Public, New York, Museum of the City of New York, 1969.

31. G. Tremblay (Éd.), Les industries de la culture et de la communication, Québec, Presses de l'Université du Québec, 1990 ; M. Raboy, I. Bernier, F. Sauvageau, D. Atkinson, Développement culturel et mondialisation de l'économie, Québec, Institut québécois de recherche sur la culture, 1994 ; H.l. Schiller, Culture Inc., New York, Oxford University Press, 1989. 
32. To Improve Learning. A Report to the Président of the United States by the Commission on Instructional Technology, Committee on Education and Labor House of Représentatives, U.S. Government Printing Office, Washington, mars 1970.

33. To Improve Learning définit ainsi la technologie éducationnelle: «a systematic way of designing, carrying out, and evaluating the total process of learning and teaching in terms of specifie objectives, based on research in human learning and communication, and employing a combination of human and nonhuman resources to bring about more effective instruction. » To Improve Learning, op. cit., 1970, ch. Il, p. 19.

34. To Improve Learning, 1970, op. cit., ch. II, 1970, traduction libre, passim.

35. Il n'est pas possible ici de rapporter même brièvement la contribution de l'évaluation muséale. Pour un survol, consulter: Muséum international, Paris, UNESCO, 1993, num. 178 (vol. $\mathrm{XLV}$, num. 2). Pour les intéressés, plusieurs bibliographies sont disponibles.

36. L'Exploratorium accueille un peu moins de 650000 visiteurs par an : $74 \%$ sont des adultes, $36 \%$ des enfants et des adolescents de moins de 18 ans auxquels il présente 600 expérimentations sur une surface de $8000 \mathrm{~m}^{2}$. Le budget annuel de fonctionnement s'élève à $7792 \mathrm{~K}$ dollars (exercice 1990-1991). Les entrées générales représentent $21 \%$ des revenus du musée, les événements spéciaux $2 \%$, les membres $4 \%$, les boutiques et la cafétéria $9 \%$, les services de consultation et de location du musée $15 \%$, les dons privés $29 \%$ et les revenus publics $19 \%$ (État de Californie, Fondation nationale pour la science, Département fédéral de l'éducation, taxe sur les hôtels, etc.). Environ $50 \%$ du revenu provient donc de ses propres ventes. Source: The Exploratorium, Tacts and Figures, janvier 1991.

37. G. Delacôte, Savoir apprendre. Les nouvelles méthodes, Paris, Odile Jacob, 1996, p. 90.

38. G. Delacôte, op. cit., 1996, p. 86.

39. Imprégné de philosophie californienne, l'Exploratorium, né dans l'effervescence du mouvement de la contre-culture, préconise le développement de relations informelles et non hiérarchiques. Dans sa forme comme dans son mode de fonctionnement, il évoque plus un centre de création, où se côtoient artistes, créateurs et scientifiques, qu'un véritable musée. Fait à noter: neuf scientifiques détenteurs d'un $\mathrm{Ph}$. D., sur quatre-vingt-dix-sept employés à temps plein et cent deux à temps partiel y travaillent. C'est exceptionnel ! Mais, c'est peut-être ce qui le distingue et le différencie le plus des autres musées de science américains.

40. Notons au passage que les enseignants sont rémunérés pour suivre cette formation. La moitié de leur salaire est payée par l'Exploratorium et l'autre par leur école. Ce détail a son importance, car la majorité des enseignants ne sont pas rétribués durant l'été.

41. Pour ce faire, il met du matériel pédagogique à la disposition des instituteurs comme les célèbres Cook-books, qui expliquent comment reproduire deux cents artefacts du centre, et les Snack-books conçus pour les enseignants. Il leur fallait des artefacts faciles à construire ne requérant que des matériaux simples et de petites tailles. Les cent sept artefacts décrits n'exigent que des moyens très simples pour être réalisés. De plus, les Snack-books contiennent toutes les explications de base nécessaires à la compréhension des phénomènes.

42. Voir: H. Hein, The Exploratorium, The Museum as Laboratory, Washington, London, Smithsonian Institution Press, 1990.

43. Il faudrait aussi tenir compte de l'accroissement du parc muséal. À quelques exceptions près, il a été financé par l'État. En France, par exemple, le parc muséal s'est considérablement enrichi au cours des vingt dernières années. Que l'on songe à tous les équipements dont a été gratifiée la capitale. Que l'on songe aussi au budget de fonctionnement que requiert chacun de ces équipements. À une plus petite échelle, une ville comme Montréal au Canada, de deux millions d'habitants s'est dotée en moins de dix ans d'un biodôme, d'un cosmodôme, d'un insectarium, d'une biosphère, d'un musée d'archéologie (Pointe-à-Callière), d'un musée d'Art moderne, d'un musée de la biologie et des biotechnologies (Armand-Frappier), d'un musée des Hospitalières, d'un agrandissement majeur du musée des Beaux-Arts, de celui du musée de l'histoire 
canadienne (McCord), et, ce, en excluant les institutions déjà existantes qui ont été rénovées. Cet accroissement n'est pas propre à la France ni au Canada, on l'observe en Europe et dans toute l'Amérique. Pour un moribond, que stigmatisait Dagognet, le musée avait encore quelques belles années devant lui. Certes la question de la patrimonialisation occupe une place de plus en plus importante dans les débats contemporains et l'accent mis sur le parc muséal est certainement un élément de réponse. On peut aussi se demander dans quelle mesure la présence accrue de l'État (immobilisations et fonctionnement d'un nombre accru d'institutions) n'est pas un facteur d'homogénéisation des pratiques. Il faudrait aussi tenir compte du développement systématique d'une industrie touristique soucieuse de plus en plus de capitaliser sur des équipements culturels. Malheureusement, l'espace limité mis à notre disposition ne nous permet pas d'aller plus avant.

44. Voir : OCDE, Perspectives économiques de l'OCDE, décembre 1994, Annexe A.

45. M. Gibbons, C. Limoges, H. Nowotny, S. Schwartzman, P. Scott. M. Trow, The New Production of Knowledge, London, Sage, 1994, passim ; C. Limoges, L'université entre la gestion du passé et l'invention de l'avenir, Symposium de la commission de planification, Université du Québec, ronéotypé, 1995, passim.

46. C. Limoges, op. cit., p. 14 et suivantes, 1995, passim.

47. Les intéressés peuvent obtenir de l'information sur ce projet en composant directement: $<$ http://sln.fi.edu>.

48. S. Beaumann, « Science Museums on the Net », Museum News, May/june 1995, p. 46.

49. S. Beaumann, «We wanted more. We needed more. The Franklin Institute is not just about access to science - it is about participation in science. Our exhibits are not just text. They are sounds, pictures, video, interactivity. We are more about questions than answers and believe that the process of searching for a solution is as valuable as finding a solution ", op. cit., 1995, traduction libre.

50. Notons au passage que le Franklin Institute Science Museum est visité depuis décembre 1994 par environ 40000 cybernautes chaque semaine. S. Beaumann, op. cit., 1995, p. 69.

51. S. Beaumann, op. cit., 1995, passim.

52. P. M. Helfrich, Building Onramps to the Information Superhighway: Designing, Implementing, and Using local Muséum Infrastructure, Philadelphie, Franklin Institute Science Muséum, v.1.2, 1995, $<$ http://sln.fi.ed/ helfrich/onramps.html>.

53. Ces travaux se développent selon deux axes: celui de l'évaluation traditionnelle dont l'objectif ultime est la satisfaction du visiteur et la compréhension du message véhiculé par l'exposition; celui de l'étude des conditions d'apprentissage dans un contexte non formel. Voir notamment: A. Giordan, G. Vecchi (de), Les origines du savoir, Neuchâtel, Delachaux \& Niestlé, 1987 ; A. Giordan, C. Souchon, M. Cantor, Évaluer pour innover. Musées, Médias et Écoles, Nice, Z'éditions, 1993 ; M. Borun, Measuring the Immeasurable. A Pilot Study of Museum Effectiveness, Philadephie, The Franklin Institute Science Museum \& Planetarium, 1977, ronéotypé; et pour synthèse des travaux, D. Jacobi, B. Schiele, «La vulgarisation scientifique et l'éducation non formelle », Revue française de pédagogie, n 91, avril-mai-juin 1990, p. 81-111.

54. Voir : G. Delacôte, op. cit, 1996.

55. Pour reprendre la belle expression de S. Moscovici. 


\section{RÉSUMÉS}

À la fois lieu de médiation et de négociation d'informations destinées au public, les musées scientifiques qui ont connu de nombreuses évolutions doivent une fois de plus s'adapter aux progrès technologiques en prenant en compte les nouveaux lieux de production des connaissances qui permettront une nouvelle dynamique école/musée.

\section{INDEX}

Index géographique : France

Mots-clés : éducation non-formelle, éducation scientifique, musée, sciences, curriculum

\section{AUTEUR}

\section{BERNARD SCHIELE}

Département des communications, Centre interuniversitaire de recherche sur la science et la technologie UQUAM, co-directeur du GRHITE, Québec, Canada. 